\title{
Comment on "EURACAN/IASLC proposals for updating the histologic classification of pleural mesothelioma: towards a more multidisciplinary approach"
}

\author{
Wickii T. Vigneswaran, Muaiad Kittaneh \\ Department of Thoracic and Cardiovascular Surgery, Loyola University Health System, Maywwod, IL, USA \\ Correspondence to: Wickii T. Vigneswaran, MD, MBA, FACS. Professor and Director of Thoracic Surgery, Department of Thoracic and \\ Cardiovascular Surgery, Loyola University Health System, System Director of Thoracic Surgery, Edward-Elmhurst Health, Building 110 Suite 6256, \\ 2160 S First Avenue, Maywood, IL 60153, USA. Email: Wickii.Vigneswaran@lumc.edu. \\ Comment on: Nicholson AG, Sauter JL, Nowak AK, et al. EURACAN/IASLC proposals for updating the histologic classification of pleural \\ mesothelioma: towards a more multidisciplinary approach. J Thorac Oncol 2020;15:29-49.
}

Submitted Mar 27, 2020. Accepted for publication Jun 10, 2020.

doi: 10.21037/tlcr-2020-14

View this article at: http://dx.doi.org/10.21037/tlcr-2020-14

The authors ought to be congratulated on standardizing pathological classification detailed analysis of currently available evidence of the pleural mesothelioma (1). They have reviewed the importance of adequate tissue sampling, and pathology reporting of features that include architecture, nuclear grading and histological characteristics that are important prognostic markers. These are important in the diagnosis and management considerations in mesothelioma. At present, it is not uncommon for patients to be referred to specialized centers where the pathology reporting of sampled tissue is suboptimal and the basic details are lacking which makes arriving at management decisions more challenging. This proposal hopefully will help and set some expectations of detailed pathological reporting to provide the clinicians with adequate information that are necessary for management decisions. This proposal further emphasizes why it is important that the patients with mesothelioma should be managed in specialized mesothelioma centers to allow proper diagnostic evaluation, pathologic classification and treatment decisions. The authors had attempted to identify histologic and molecular biomarkers that are prognostic in nature so that we identify patients with more aggressive disease. Establishing predictive pathologic information that inform treatment decisions is still a subject of research. There are ongoing efforts in searching for actionable biomarkers in mesothelioma but there has been no level 1 evidence or FDA approved therapies for this fatal disease yet (Table 1).
$B A P 1$ germline mutations that result in loss of function of BAP1 protein seem to inform a better overall prognosis and response to platinum-based chemotherapy in mesothelioma patients. A study published by Hassan et al. suggested that patients with pleural mesothelioma with lossof-function mutations in BAP1 and other DNA repair genes appeared to benefit from platinum chemotherapy compared with patients without inherited mutations (2).

The Mesothelioma Stratified Therapy Trial (MiST) is a multi-drug Phase II Trial in Malignant Mesothelioma that is exploring the benefit of Rucaparib in BRCA1/ BAP1 mutant mesothelioma, Abemaciclib in p16INK4A negative mesothelioma, Atezolizumab \& Bevacizumab in PD-L1 positive mesothelioma patient and pembrolizumab $\&$ bemcentinib in patients with No specific biomarker (NCT03654833). More collaborative efforts among the mesothelioma experts are required to expedite enrollment in global trials that could bring some guidance.

Biomarker driven clinical trials are strongly needed because the traditional histologic classification has not made significant change to the current standards in treating mesothelioma for the last 2 decades (Figure 1). We agree that a multidisciplinary approach with more specific histopathologic and biomarker identification is essential for better understanding of the disease but a more collaborative approach to identify these patients and enroll them in global clinical trials will help patients, physicians and scientist to answer these questions more rapidly. We 
also believe that access to real world data that incorporates relevant biomarker information in all patients and treatment outcomes could expedite reclassification of mesothelioma not only on the basis of histologic features but also on the basis of current trends in cancer care using molecular biomarkers. We congratulate the multi institutional contributor to this valuable and practical standardization of histological classification of pleural mesothelioma.

Table 1 Current list of BAP1 loss and NF2 mutant biomarker driven clinical trials in mesothelioma

\begin{tabular}{lllllll}
\hline Sponsor & Product & MOA & Phase & Biomarker & Country & NCT\# \\
\hline NCI & Olaparib & PARB inhibitor & II & BAP1 loss & US & NCT03531840 \\
Univ of Florida & Niraparib & PARB inhibitor & II & BAP1 loss & US & NCT03207347 \\
Epizyme Inc. & Tazemetostat & EZH2 inhibitor & II & BAP1 loss & US & NCT02860286 \\
MSK/Takeda & Pevonedistat (MLN4924) & NEDD8 inhibitor & I/II & NF2 mutant & US & NCT03319537 \\
Verastem Oncology & VS-6063 (Defactinib) & FAK inhibitor & I/II & NF2 mutant & US & NCT02004028 \\
NHS/Nerastem/Merck \& Co. & VS-6063 (Defactinib)/Pembrolizumab & FAK inhibitor + PD1 I/II & NF2 mutant & UK & NCT02758587 \\
\hline
\end{tabular}

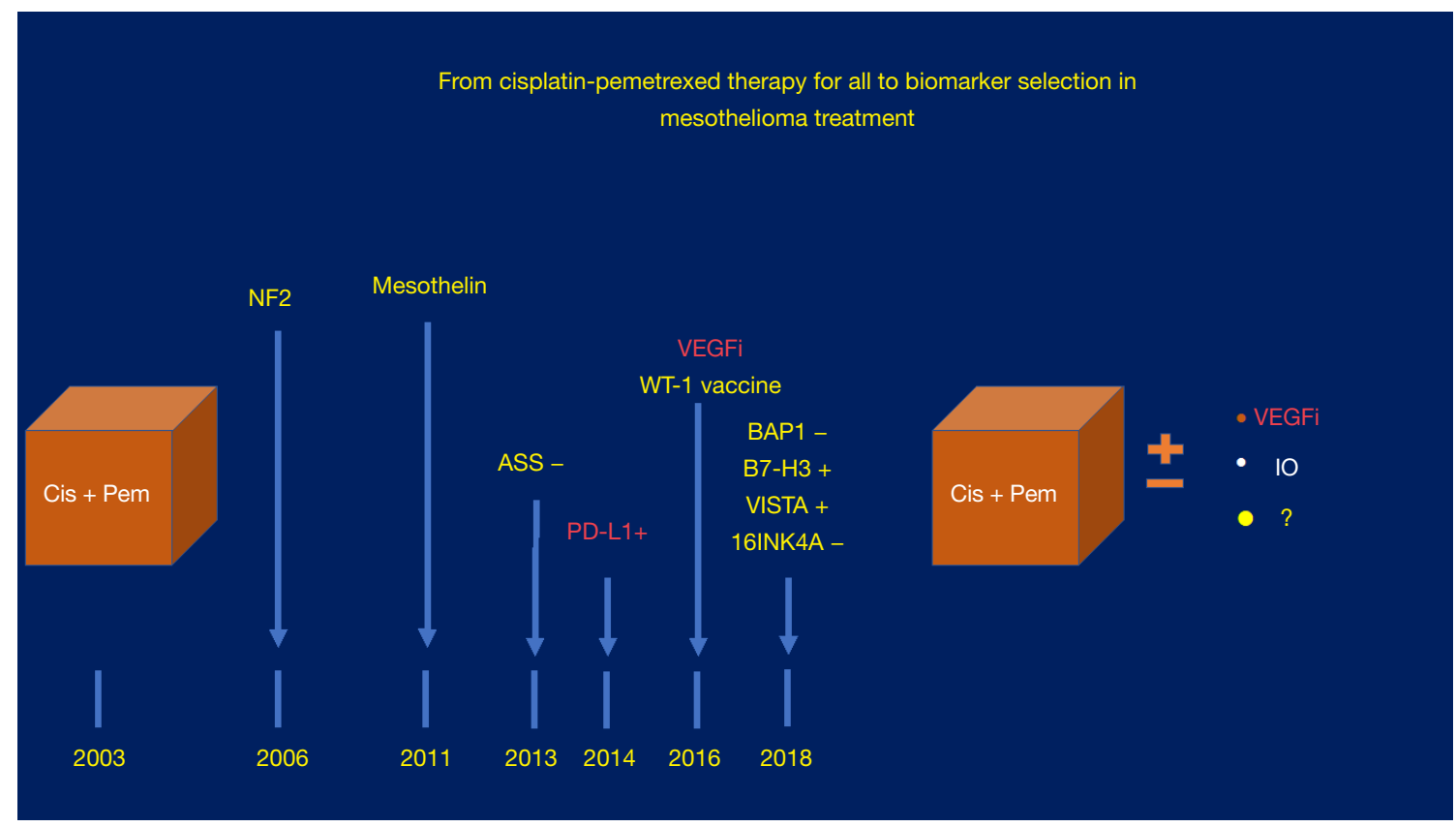

Figure 1 Historical timeline in search of actionable targets in mesothelioma. Cis, cisplatin; Pem, pemetrexed; NF, neurofibromatosis; ASS, arginosuccinate synthetase; PD-L1, programmed death receptor ligand 1; WT-1, Wilms tumor; VEGF, vascular endothelial growth factor; VISTA, set immunoregulatory receptor antibody; $\mathrm{p} 16^{\text {Ink4a }}$, the principal member of the Ink4 family of CDK inhibitors; IO, immunotherapy.

\section{Acknowledgments}

Funding: None.

\section{Footnote}

Provenance and Peer Review: This article was commissioned by the editorial office, Translational Lung Cancer Research. The article did not undergo external peer review.

Conflicts of Interest: Both authors have completed the ICMJE uniform disclosure form (available at http://dx.doi. org/10.21037/tlcr-2020-14). Both authors have no conflicts 
of interest to declare.

Ethical Statement: The authors are accountable for all aspects of the work in ensuring that questions related to the accuracy or integrity of any part of the work are appropriately investigated and resolved.

Open Access Statement: This is an Open Access article distributed in accordance with the Creative Commons Attribution-NonCommercial-NoDerivs 4.0 International License (CC BY-NC-ND 4.0), which permits the noncommercial replication and distribution of the article with the strict proviso that no changes or edits are made and the original work is properly cited (including links to both the formal publication through the relevant DOI and the license). See: https://creativecommons.org/licenses/by-nc-nd/4.0/.

\section{References}

1. Nicholson AG, Sauter JL, Nowak AK, et al. EURACAN/ IASLC proposals for updating the histologic classification of pleural mesothelioma: towards a more multidisciplinary approach. J Thorac Oncol 2020;15:29-49.

2. Hassan R, Morrow B, Thomas A, et al. Inherited predisposition to malignant mesothelioma and overall survival following platinum chemotherapy. Proc Natl Acad Sci U S A 2019;116:9008-13.
Cite this article as: Vigneswaran WT, Kittaneh M. Comment on "EURACAN/IASLC proposals for updating the histologic classification of pleural mesothelioma: towards a more multidisciplinary approach". Transl Lung Cancer Res 2020;9(6):2508-2510. doi: 10.21037/tlcr-2020-14 